\title{
Suppression and retinal correspondence in intermittent exotropia
}

\author{
JEFFREY COOPER AND CAROL DIBBLE RECORD \\ From the Institute of Vision Research, SUNY/State College of Optometry, 100 East 24th Street, New York, \\ New York 10010, USA
}

SUMMARY Suppression scotomas and retinal projection (retinal correspondence) were measured in six intermittent exotropes during deviation. Measurements used red-green anaglyph stimuli presented on a black background which could be varied from 3.4 minutes of arc to $3^{\circ} 24^{\prime}$. Results showed non-suppression of all points between the fovea and the diplopia point. Harmonious anomalous retinal correspondence was usually observed. Two subjects had spontaneous changes from anomalous retinal correspondence to normal retinal correspondence without a concurrent change in ocular position. Conventional testing resulted in more variable results in regard to retinal correspondence and suppression, suggesting that non-suppression and anomalous retinal correspondence occur when black backgrounds are used for testing.

Patients with intermittent exotropia of the divergence excess type (DE) or basic exotropia usually do not complain of diplopia when their eyes are deviating.' Parks ${ }^{2}$ believes that the absence of diplopia is due to a dense temporal hemiretinal suppression. Using a technique employing Risley prisms, Jampolsky $^{3}$ demonstrated that DE patients have a suppression scotoma extending from the fovea to the point on the retina of the deviating eye which projects to the object of regard (point zero or diplopia point). Travers ${ }^{4}$ and Pratt-Johnson and Wee ${ }^{5}$ measured dense suppression scotomas at the fovea and point zero of the deviating eye in intermittent exotropes. Awaya et al. ${ }^{6}$ measured suppression at the fovea and point zero in 11 of 25 of the intermittent exotropes that they tested, while using first degree target projected on a light fusional background.

The clinically accepted convention is that exotropes have a dense suppression in the deviating eye. There is, however, evidence suggesting that intermittent exotropes do not always suppress the deviating eye. First, DE patients can often experience diplopia when a red lens is placed over the fixating eye, while viewing a muscle light in a darkened room. Secondly, Cooper and Feldman ${ }^{7}$ have demonstrated non-suppression of the fovea in the deviating eye of $\mathrm{DE}$ patients. Thirdly, DE patients often have non-suppression of the afterCorrespondence to Jeffrey Cooper, OD. image and anomalous retinal correspondence (ARC) in the deviated position and normal retinal correspondence (NRC) in the aligned position on the Hering-Bielschowsky afterimage test. ${ }^{8}$

We therefore attempted to qualify the depth of suppression in deviating intermittent exotropes while monitoring ocular position. We used variable first degree anaglyph targets to measure suppression scotomas. In the event of suppression we attempted to break the suppression and measure the retinal correspondence between point zero and the fovea of the deviating eye.

\section{Subjects and methods}

There were six subjects, four were female and two male. All had intermittent exotropia as measured by a distance and near cover test. Each was capable of performing in a restrained head apparatus and could give valid and reliable responses. They all had visual acuities correctable to at least $20 / 20$ in each eye and could perceive at least 40 arc seconds of stereopsis on the Randot stereo test when binocularly aligned.

\section{APPARATUS}

An Atari 800 personal computer was used to present anaglyph stimuli on a Sony 19 -inch $(48-\mathrm{cm})$ Color Monitor. ${ }^{9}$ The patient was seated $40 \mathrm{~cm}$ from the monitor and wore Keystone red-green glasses. The 
red lens was in front of the habitually fixating eye. The monitor displayed a red cross, which subtended an angle of $2^{\circ}$; each line of the cross had a width of 3.9 minutes of arc. The cross, viewed by the normally fixating eye, had a mean luminance of $2 \mathrm{~cd} / \mathrm{m}^{2}$. The second target was a green movable square or rectangle which also had a mean luminance of $2 \mathrm{~cd} / \mathrm{m}^{2}$. The green target could be instantly changed from a single-dot pixel (3.4 minutes of arc) to a 60 -dot by 30 -dot horizontal rectangle. The maximum green shape subtended an angle of $\left(3^{\circ} 24^{\prime} \times 1^{\circ} 42^{\prime}\right)$ to the patient. The green target was movable so as to measure suppression areas. The background was black. The red and green targets were such that the red target could be seen only by the eye wearing the red filter and the green target by the eye wearing the green filter. Eye movements were monitored with a Biotronics Infrared Eye system combined with an auditory biofeedback apparatus. Thus the examiner was assured of stable deviation or alignment during testing. Ocular movements more than $1^{\circ}$ from the present position (i.e., alignment or measured deviation position) resulted in an auditory tone (Fig. 1).

\section{METHODS}

The following conventional clinical tests were performed: Randot stereo test; troposcope evaluation of subjective and objective angles with a $1^{\circ}$ target; Hering-Bielschowsky afterimage test; Bagolini striated lens test; and a red lens test for diplopia. All afterimage testing, Bagolini lens tests, and red lens tests were done in a darkened room. All ARC testing was done in both the aligned and deviated position.

Suppression areas and retinal correspondence were measured and recorded with the Atari 800 microcomputer and a colour TV monitor. The patient fixated the red cross while the examiner moved the green target. Initially the examiner determined the objective angle of deviation by means of occlusion (i.e., cover test). The green target was moved until no movement on the cover test was observed. The eye movement apparatus was then

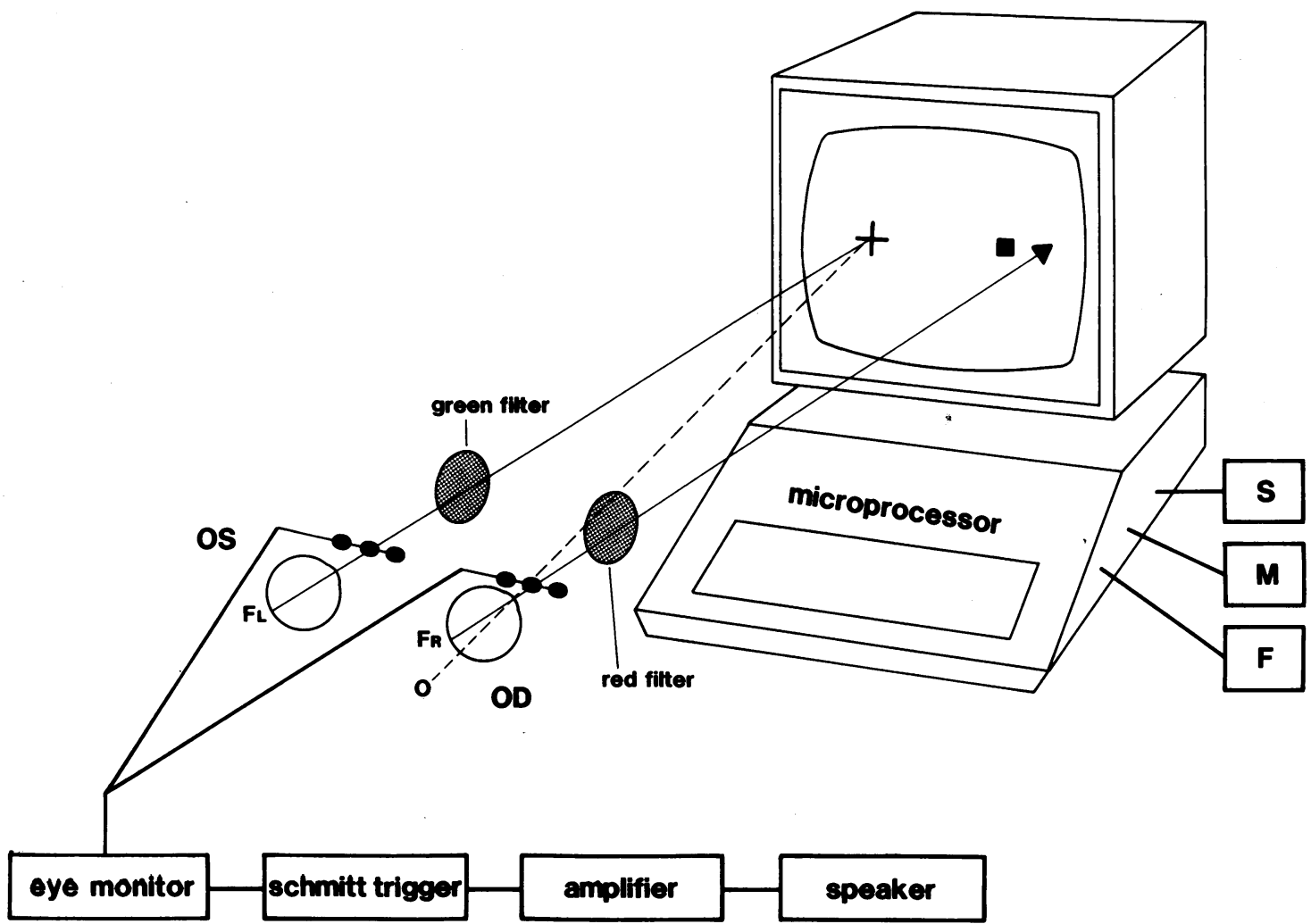

Fig. 1 Patient is a right exotrope wearing red-green anaglyph glasses and infrared eye movement glasses. While viewing a colour monitor $40 \mathrm{~cm}$ away the patient fixates a red cross (+) with the $O S$ and the examiner moves green box (D) seen by the $O D$. Examiner may control stimulus size with controller marked $\mathrm{S}$; move stimulus with $\mathrm{M}$; or move the fixation target with $\mathrm{F}$. $\mathrm{F}_{\mathrm{R}}$ is the fovea of the $\mathrm{OD} ; \mathrm{F}_{\mathrm{L}}$ is the fovea of the $\mathrm{OS} ;$ and $\mathrm{O}$ is point zero or the diplopia point. Ocular position during binocular perimetric evaluation is monitored with Biotronics eye movement monitor system. 
calibrated so that, when the left eye moved from its position, a tone was triggered, and when the right eye deviated a click was heard. The patient was then asked to describe the relative position of the red and green targets. He/she was also asked to report if either the red or green targets disappeared. The examiner then moved the green target until the patient reported superimposition (i.e., subjective angle).

The green target was moved through the visual field; the patient was asked to report any disappearance of either target. If the green target disappeared, the examiner used standard perimetric techniques to map out the scotoma. Larger targets were produced to determine the density of the suppression scotoma. Plots were done horizontally, vertically, and obliquely. Randomly placed points were also presented to pick up small scotomas and to verify kinetically determined retinal projection. Suppression areas were recorded by the examiner by depressing a button on the joystick controller.

Periodically during testing the patient was asked to point to either the fixation or the test target and to tell the examiner if either target ever disappeared. The patient was also asked to describe the relative position of each target. The examiner used 'catch trials' by extinguishing the stimulus to assure the validity of the patient responses.

\section{Results}

Table 1 presents both experimental and orthoptic findings in the six intermittent exotropes tested. Five of the six subjects showed no suppressions during experimental testing, even with the smallest possible target. The sixth patient suppressed all size targets throughout experimental testing. Four of the nonsuppressing patients gave ARC responses, with one showing NRC. Two of the ARC responders (nos. 1

Table 1 Orthoptic and experimental findings

\begin{tabular}{llllll}
\hline \multicolumn{3}{c}{$D V$} & $N V$ & & \\
$P T$ & $C T$ & $C T$ & \multicolumn{1}{l}{$T R O P E X P$} & BAG HBD HBA RL \\
\hline 1 & 30 & 20 & Sup & NRC/ARC ARC ARC NRC Sup \\
2 & 20 & 14 & Sup & HARC & ARC NRC NRC Sup \\
3 & 45 & 50 & Sup & NRC/ARC Sup NRC NRC Paradoxial dip \\
4 & 57 & 59 & NRC & NRC & NRC NRC NRC NRC \\
5 & 25 & 18 & Sup & ARC & Sup ARC NRC NRC \\
6 & 50 & 12 & Sup & Sup & Sup ARC NRC Sup \\
\hline
\end{tabular}

SUP $=$ suppression. $C T=$ prolonged cover test distance $(D V)$ and near (NV) in prism dioptres to obtain maximum deviation.

TROP $=$ troposcope findings at the objective angle. $E X P=$ red-green anaglyph findings during experimental testing. BAG=Bagolini lens results while an eye deviates. $\mathrm{HBD}=$ Hering-Bielschowsky test results during deviation. $\mathrm{HBA}=$ Hering-Bielschowsky test results during alignment. $R L=$ red lens test results during deviation. and 3) briefly changed from harmonious ARC to NRC without a concurrent change in ocular position during the course of testing. These two subjects usually showed ARC. One patient (no. 4) showed NRC responses throughout testing and had the largest deviation in the study on cover test.

Standard clinical testing yielded more varied results. In the troposcope with first degree targets on a light background, five subjects suppressed completely and one showed NRC. Bagolini striated lens testing during deviation resulted in ARC responses for two subjects, NRC in one subject, and suppression for the remaining three subjects. The HeringBielschowsky afterimage test performed in a darkened room yielded three cases of ARC and three of NRC with no suppressions reported.

\section{Discussion}

Our results indicated the presence of non-suppression of luminous targets in five of the six patients across the temporal portion of the retina during experimental testing. ARC was found during deviation and NRC during alignment. These findings support Morgan's motoric theory of ARC. ${ }^{10}$ However, neither Burian's" adaptation theory nor Morgan's theory are useful in explaining the instantaneous changes in correspondence without a concurrent change in ocular position observed in two patients in this study and in a previous study. '

The Hering-Bielschowsky and Bagolini lens tests which are used to determine the presence of NRC were done in a darkened room. The HeringBielschowsky test indirectly determines the presence of foveal suppression in either eye, while the Bagolini test indicates the presence of suppression at 'zero point'. It was shown that there was more suppression on the Bagolini test than on the Hering-Bielschowsky test, indicating that the zero point (diplopia point) of the deviating eye suppresses more readily than the fovea of the deviating eye. The clinical observation that it is more difficult to suppress the afterimages of the Hering-Bielschowsky test than the streaks of the Bagolini test in a darkened environment was confirmed by our study.

Our findings are consistent with Jampolsky's observation that not only size and brightness determine the extent of suppression. We found that colour complexity of the stimulus and similarity of targets presented to the two eyes may change suppression characteristics. Only one patient suppressed during our experimental procedure, which used different coloured stimuli on a black background. PrattJohnson and Wee ${ }^{5}$ found both suppression of the fovea in the deviating eye and suppression of the deviating eye at the point matched to the fovea of the 
fixating eye (zero point). We believe that Travers's ${ }^{4}$ use of non-illuminated targets and Pratt-Johnson and Wee's' use of a white background triggered suppressions and resulted in findings different from ours.

Awaya et al. ${ }^{6}$ used a phase difference haploscope with and without a fusible background. Of the 11 intermittent exotropes tested, who had a manifest deviation during testing, nine showed suppression at point zero when a fusible background was present. Without a fusible background 15 showed suppression of the deviating fovea, and the remaining 10 showed superimposition at the objective angle. They concluded that intermittent exotropes have suppressions with NRC. We did not find the consistency of NRC nor the suppression that Awaya et al. reported. ${ }^{6}$ These findings which differ from ours may be explained by the observation of Jampolsky ${ }^{3}$ and Griffen $^{12}$ that differences in testing apparatus will alter suppression patterns.

Our findings suggest that the intermittent exotrope does not always suppress and usually has ARC under optimal conditions, even with very small stimuli. As previously stated, suppressions are dependent on the background. Black backgrounds seem to eliminate suppressions, while light backgrounds result in active inhibition or suppression. Our patients showed harmonious ARC, which extended from the point zero past the fovea and through the entire nasal retina. Like suppressions, the ARC is liable to change under certain test conditions. Retinal correspondence changes with ocular position and occasionally without any change in stimuli or eye movements.

These results may have important implications in the orthoptic treatment of intermittent exotropia. If techniques can be employed which disrupt both the anomalous retinal correspondence mechanism and the suppression mechanism, the DE patient will find it difficult to maintain deviation in the presence of NRC diplopia. Sanflippo and Clahane, ${ }^{13}$ Cooper, ${ }^{1}$ and Awaya et al. ${ }^{6}$ have advocated diplopia awareness and antisuppression therapy in the treatment of intermittent exotropia. Our study suggests that one should start therapy with different coloured targets presented to each eye with a darkened background. Therapy continues by gradually increasing the ambient light and decreasing the filter intensity until the DE patient can experience NRC diplopia without the use of filters in a fully illuminated room. In this way one can be sure of breaking not only suppressions but ARC patterns as well.

In summary, our intermittent exotropes of the DE type did not show suppressions even with very small targets (3.4 minutes of arc.) at either the fovea or diplopia point of the deviating eye. Most of our patients showed harmonious ARC from the diplopia point to the fovea. Both suppression and retinal correspondence seem to change in accordance with stimuli, background, and retinal stimuli. Our results, which conflict with those of previous authors, are probably explained by accurate control of ocular position and use of a dark, non-fusible background with dissimilar targets. Our results suggest nonstability of a retinal correspondence (usually ARC) and non-suppression in the intermittent exotrope of the divergence excess type during deviation with small luminous targets on a dark background.

\section{References}

1 Cooper J. Intermittent exotropia of the divergence excess type. J Am Optom Assoc 1977; 48: 1261-63.

2 Parks M. Concomitant exodeviations. In: Duane T, ed. Clinical ophthalmology. Philadelphia: Harper and Row, 1983; 1.

3 Jampolsky A. Characteristics of suppression in strabismus. Arch Ophthalmol 1955; 54: 683-96.

4 Travers TB. Suppression of vision in squints and its association with retinal correspondence and amblyopia. $\mathrm{Br} J$ Ophthalmol 1938; 22: 577-604.

5 Pratt-Johnson J, Wee HS. Suppression associated with exotropia. Can J Ophthalmol 1969; 4: 136-44.

6 Awaya S, Nozaki H, Itoh T, Kikuko H. Studies of suppression in alternating constant exotropia. In: Moore S, Mein S, Stockbridge L, eds. Orthoptics: Past, Present and Future New York: Stratton Intercontinental: 1975.

7 Cooper J, Feldman J. Panoramic viewing, visual acuity of the deviating eye and anomalous retinal correspondence in intermittent exotropia of the divergence excess type. Am J Optom Physiol Opt 1979; 56: 422-9.

8 Bielschowsky A. Divergence excess. Arch Ophthalmol 1934; 12: 157.

9 Cooper J, Citron M. Microcomputer produced anaglyphs for evaluaton and therapy of binocular anomalies. J Am Optom Assoc 1983; 54: 785-8.

10 Morgan MW. Anomalous correspondence interpreted as a motor phenomenon. Am J Optom Physiol Opt. 1961;38: 131-48.

11 Burian HM. The sensorial retinal relationship in concomitant strabismus. Arch Ophthalmol 1947; 37: 336-68.

12 Griffen JR. Binocular anomolies procedures for vision therapy (c) 1976. Chicago, Ill. p.46.

13 Sanflippo S, Clahane A. Effectiveness of orthoptics alone in selected cases of exodeviation: the immediate results and several years later. Am Orthopt J 1970; 20: 104-17.

Accepted for publication 31 December 1985. 